\title{
Análise da Influência de Grupos de Inclusão de Mulheres em Comunidades de Software Livre
}

\author{
Mariana Prudêncio $^{1}$, Ana Paula Chaves ${ }^{1}$ \\ ${ }^{1}$ Departamento Acadêmico de Computação \\ Universidade Tecnológica Federal do Paraná - Câmpus Campo Mourão \\ Caixa Postal 271 - 87301-899 - Campo Mourão - PR - Brasil \\ mari.mvargas@gmail.com, anachaves@utfpr.edu.br
}

\begin{abstract}
Research shows a small participation of women in Open Source Software (OSS) Projects. In response to this, a number of initiatives for inclusion and retention has been created in OSS communities in order to change this disproportionality scenario. This ongoing research investigates the influence of these initiatives with regard to engagement and Psychological Sense of Community (PSC) of the women who are OSS communities' members.

Resumo. Pesquisas mostram que a participação de mulheres em software livre é baixa. Como resposta, diversas iniciativas para inclusão e retenção tem sido criadas nas comunidades de software livre com a finalidade de mudar este cenário de desproporcionalidade. Essa pesquisa em andamento aborda a influência dessas iniciativas no que diz respeito ao engajamento e sentimento psicológico de comunidade (SPC) das mulheres participantes de comunidades de software livre.
\end{abstract}

\section{Introdução}

A baixa representatividade de mulheres no ramo de tecnologia é uma realidade. No ano de 1983, as mulheres compunham cerca de $43 \%$ da força de trabalho em tecnologia da informação, de acordo com dados da U.S. Bureau of Labor Statistics Current Population Survey [Coder et al. 2009]. Entretando, em 2008, mesmo que a força de trabalho tenha mais que duplicado, o percentual de mulheres caiu para cerca de $25 \%$ [Coder et al. 2009]. Tratando de software livre, de acordo com [Ghosh et al. 2002], em 2002 menos de 1,5\% dos colaboradores eram mulheres. O cenário não mudou: de acordo com o relatório da UNESCO, publicado em 2012, o percentual de mulheres participantes de software livre era de apenas $2 \%$ [UNESCO 2012].

A preocupação com a ausência de mulheres em software livre não é apenas inclusiva uma vez que, em uma sociedade baseada em TICs (Tecnologias de Informação e Comunicação), o software é produzido para o uso de ambos os sexos [Nafus et al. 2006]. Portanto, é essencial que se conheça os requisitos para atender os mais diversos tipos de usuários. Além disso, a diversidade de desenvolvedores leva ao desenvolvimento de melhores tecnologias [Powell et al. 2010].

[Lin 2005] pontua uma série de dilemas que impedem mulheres de colaborarem efetivamente com software livre. Um dos dilemas trata da falta de modelos femininos a serem seguidos em uma comunidade. Uma vez que o percentual de mulheres em comunidades de software livre é baixo, é difícil para novatas enxergarem as mulheres presentes na comunidade. 
Para minimizar este problema, uma das soluções mais utilizadas é a criação de grupos de apoio e inclusão direcionados para mulheres. [Haché et al. 2011] justifica o fenômeno de criação desses grupos como uma iniciativa para que mulheres se sintam mais confortáveis em comunidades de software livre. Além disso, grupos tendem a destacar as contribuições realizadas pelas mulheres e consequentemente dão maior visibilidade às mulheres da comunidade.

Com base na observação desse fenômeno, este trabalho parte da hipótese de que grupos de mulheres em comunidades de software livre exercem influência sobre o Sentimento Psicológico de Comunidade (SPC) das participantes. O SPC se trata do sentimento de fazer parte de uma rede de suporte mútuo, de que se pode depender e que está sempre disponível [Sarason 1977]. Dessa forma, acredita-se que os grupos de mulheres em comunidades de software livre auxiliam na inclusão e retenção de mulheres nessas comunidades, uma vez que age como uma entidade de apoio, aumentando seu vínculo com a comunidade. Entretanto, não foram encontradas referências que tratem da influência desses grupos nas comunidades de software livre.

Para analisar esta hipótese, o objetivo desta pesquisa em andamento é responder à questão "Grupos de inclusão e retenção de mulheres exercem influência sobre o engajamento elou sentimento de pertença de mulheres em comunidades de software livre?”. Para isso, propõe-se uma pesquisa quali-quantitativa que será respondida por mulheres que participam de comunidades de software livre. A pesquisa divide as comunidades de software livre em dois grupos: Grupo 1, formado por comunidades que não possuem uma iniciativa formal para inclusão/retenção de mulheres; e Grupo 2, formado por comunidades que possuem uma iniciativa formal para inclusão/retenção de mulheres. O objetivo de delimitar esses dois grupos é comparar o SPC das mulheres pertencentes a cada grupo, visando encontrar evidências empíricas da influência que esses grupos exercem sobre o SPC.

O restante do trabalho está organizado da seguinte maneira: a seção 2 apresenta o método de pesquisa que será utilizado para atingir o objetivo preestabelecido e comprovar a hipótese. A seção 3 apresenta as ações já realizadas bem como as futuras, assim como o que se espera obter de resultados. Por fim, na seção de referências, as referências utilizadas neste trabalho.

\section{Método de Pesquisa}

Para responder à questão de pesquisa "Grupos de inclusão e retenção de mulheres exercem influência sobre o engajamento elou sentimento de pertença de mulheres em comunidades de software livre?", um conjunto de ações foram definidas, como pode ser visto na Figura 1.

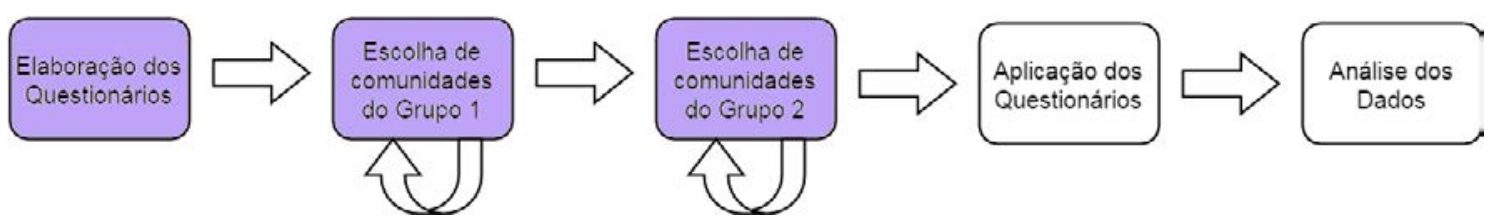

Figura 1. Fluxo de execução das ações propostas

A primeira etapa do trabalho consistiu na formulação de um questionário que in- 
daga sobre como iniciativas de inclusão auxiliam na entrada e retenção de mulheres em comunidades de software livre. O questionário foi elaborado com base no instrumento de pesquisa definido por [Obst 2004]. O questionário estará disponível em dois idiomas, inglês e português, sendo que o primeiro idioma foi escolhido por ser geralmente utilizado na comunicação em comunidades de software livre, o que aumenta a abrangência da pesquisa e o segundo idioma foi escolhido por se tratar da língua nativa dos pesquisadores.

O questionário está dividido em quatro partes. A primeira visa obter dados do perfil da participante, contendo questões relativas à idade, escolaridade, estado civil, entre outras. A segunda parte procura saber o grau de identificação da participante em relação à seu gênero. A terceira parte procura saber o quão importante a participante considera ser membro de uma comunidade de software livre. Para a segunda e terceira parte, foi utilizada escala Likert [Likert 1932], contendo 15 afirmações para cada parte. Por fim, a quarta parte contém duas perguntas abertas, que servem como um esaço para que a participante se expresse livremente, fornecendo sua opinião de forma mais abrangente a respeito do conteúdo da segunda e da terceira parte. O questionário será disponibilizado online, em um link que será posteriormente enviado às participantes convidadas.

A segunda atividade consiste em encontrar as comunidades de software livre do Grupo 1. Para encontrar as comunidades, serão analisados projetos que estejam hospedados no GitHub ${ }^{1}$. Tal plataforma foi escolhida por possuir uma quantidade significativa de projetos hospedados. A escolha de comunidades do Grupo 1 será feita com base nos critérios a seguir:

- 1 - Comunidades consolidadas: para esse trabalho, considera-se uma comunidade consolidada aquela que possui oitenta contribuidores ou mais e que estejam hospedados na plataforma GitHub há pelo menos dois anos. É esperado que existam comunidades mais antigas do que a data de sua hospedagem no GitHub. Por esta razão, a data servirá apenas como um filtro inicial para a busca. Caso não sejam encontradas comunidades suficientes para a execução do estudo, a busca será expandida.

- 2 - Não possuir iniciativas de inclusão de mulheres: as comunidades do Grupo 1 são aquelas que não possuem grupos para inserção de mulheres em software livre, portanto, a existência ou não dessas iniciativas será investigada.

Tendo escolhido as comunidades do Grupo 1, é necessário escolher as comunidades do Grupo 2. Para encontrar essas comunidades, serão utilizados dois recursos: a plataforma GitHub e o mapa colaborativo "Mapa das Mina"2. O "Mapa das Mina" consiste em um mapa colaborativo que possui marcações sobre localizações onde existem projetos de mulheres relacionados a empreendedorismo, tecnologia, saúde entre outras áreas. Alguns dos projetos de tecnologia presente no "Mapa das Mina" são relacionados a comunidades de software livre que possuem projetos hospedados no GitHub. Logo, o mapa se torna uma ferramenta interessante para este trabalho.

As comunidades do Grupo 2 serão escolhidas utilizando o primeiro critério de escolha do Grupo 1 (comunidades consolidadas). A partir disso, será feita uma análise mais profunda das ações que visam a inclusão de mulheres em software livre presentes nessas comunidades. Após a seleção de comunidades, é necessário entrar em contato

\footnotetext{
${ }^{1}$ https://github.com/

${ }^{2}$ https://goo.gl/5RqMFQ
} 
com as mulheres que participam dessas comunidades. Essas mulheres serão convidadas a responder os questionários previamente elaborados.

Por fim, os resultados obtidos serão analisados, comparando as respostas ao questionário fornecidas pelas participantes do Grupo 1 e do Grupo 2, com a finalidade de atingir o objetivo preestabelecido. Os resultados devem fornecer evidências empíricas da influência dos grupos de inclusão/retenção no engajamento das mulheres na comunidade.

\section{Resultados Esperados}

Este trabalho é uma pesquisa em andamento que possui como objetivo analisar a influência de grupos de inclusão e retenção de mulheres em projetos de software livre, verificando se as participantes se sentem mais engajadas com a comunidade quando esta oferece iniciativas de empoderamento. No momento da escrita deste artigo, o trabalho está na fase de escolha de comunidades de software livre de interesse. Os resultados serão divulgados em publicações futuras. Para próximas ações, é necessário que o questionário seja enviado para as colaboradoras das comunidades escolhidas. Os questionários serão enviados via email, com texto personalizado para cada colaboradora, relacionando suas contribuições com a comunidade de software livre com os objetivos da pesquisa, a fim de motivá-las a responder. Após o levantamento dos dados, os mesmos serão analisados.

A principal contribuição deste artigo é descrever o método para analisar a influência das ações de inclusão e fomentar a investigação a respeito da eficácia dessas ações. Uma vez que várias ações para inclusão feminina em software livre vêm sendo criadas, espera-se que os resultados desse estudo, quando alcançados, indiquem a influência de tais ações. Espera-se também que os resultados sirvam como base para a ampliação e manutenção das ações de empoderamento de mulheres em software livre e que influencie os demais pesquisadores da área de software livre a estudar iniciativas de inclusão de mulheres nas comunidades.

\section{Referências}

Coder, L., Rosenbloom, J. L., Ash, R. A., and Dupont, B. R. (2009). Economic and business dimensions - increasing gender diversity in the IT work force. Commun. ACM, 52(5):25-27.

Ghosh, R. A., Glott, R., Krieger, B., and Robles, G. (2002). Free/libre and open source software: Survey and study. RA Ghosh, International Institute of Infonomics, University of Maastricht and Berlecon Research GmbH.

Haché, A., Cruels, E., and Vergés, N. (2011). Mujeres programadoras y mujeres hackers. uma aproximación desde lela coders.

Likert, R. (1932). A technique for the measurement of attitudes. Archives of psychology.

Lin, Y. (2005). Inclusion, Diversity and Gender Equality: Gender Dimensions of the Freellibre Open Source Software Development. IDEA Groups.

Nafus, D., Leach, J., and Krieger, B. (2006). Gender: integrated report of findings. FLOSS POLS Deliverable D, University of Cambridge.

Obst, P. (2004). Community connections:psychological sense of community and identification in geographical and relational settings. $\mathrm{PhD}$ thesis, Queensland University of Technology. 
Powell, W. E., Hunsinger, S., and Medlin, D. (2010). Gender differences within the open source community: An exploratory study. Journal of Information Technology Management, 21(4).

Sarason, S. (1977). The psychological sense of community: prospects for a community psychology. Jossey-Bass behavioral science series. Jossey-Bass.

UNESCO (2012). Unesco is looking for initiatives promoting women's role in free and open source software. 